\title{
Operative Möglichkeiten der Hyperhidrosebehandlung
}

\section{Surgical Treatment for Hyperhidrosis}

Autor

Institut

\section{F. G. Bechara}

Klinik für Dermatologie, Venerologie und Allergologie, Ruhr-Universität Bochum
Bibliografie

DOI http://dx.doi.org/

10.1055/s-0031-1291645

Online-Publikation: 31.1.2012

Akt Dermatol 2012; 38: 311-315

(c) Georg Thieme Verlag KG

Stuttgart · New York

ISSN 0340-2541

Korrespondenzadresse

Priv.-Doz. Dr. Falk G. Bechara

Leiter des Bereichs

Operative Dermatologie

Klinik für Dermatologie,

Venerologie und Allergologie

Ruhr-Universität Bochum

Gudrunstr. 56

44791 Bochum

f.bechara@klinikum-bochum.de

\section{Zusammenfassung \\ $\nabla$}

Die operative Versorgung stellt immer die Ultima Ratio in der Behandlung der Hyperhidrose dar, nachdem konservative Verfahren ausgeschöpft sind. Operationen eignen sich nur zur Therapie

\section{Einleitung \\ $\nabla$}

Hyperhidrosen sind eine heterogene Gruppe von Krankheitsbildern, die mit einer pathologisch gesteigerten Schweißsekretion einhergehen [1]. Ursächlich kann zwischen einer primären und einer sekundären Hyperhidrose unterschieden werden. Sekundäre Hyperhidrosen stellen eine interdisziplinäre Herausforderung dar, da ihnen oft internistische (z.B. Infektionskrankheiten), endokrinologische (z.B. Schilddrüsenfunktionsstörungen) oder neurologische (z.B. Plexusläsionen) Ursachen zugrunde liegen [1,2]. Die primäre Hyperhidrose, die fokal oder generalisiert auftreten kann und durch eine Überstimulation des sympathischen Anteils des vegetativen Nervensystems bedingt ist, ist hingegen ureigenes dermatologisches Gebiet. Fokale Hyperhidrosen treten vor allem axillär, palmoplantar und fazial auf und betreffen ca. $1 \%$ der westlichen Bevölkerung [3]. Die Erkrankung kann mit einer stark reduzierten Lebensqualität einhergehen und erhebliche soziale und berufliche Einschränkungen zur Folge haben [4].

\section{Diagnose}

Eine primäre fokale Hyperhidrose wird klinisch diagnostiziert ( Tab.1). Der Schweregrad kann anhand eines semiquantitativen klinischen Scores erhoben werden ( Tab.2) [2]. Einfacher in der Routine anwendbar ist eine Skala mit 4 Punkten zur Bestimmung des Schweregrades der primären Hyperhidrose (Hyperhidrosis Disease fokaler Hyperhidrosen, wobei axillär lokal chirurgische Maßnahmen möglich sind, während palmar eine endoskopische Sympathektomie zum Einsatz kommen kann. Leider fehlen für viele operative Verfahren evidenzbasierte Daten bezüglich Effektivität und Langzeitergebnissen.

Severity Scale/HDSS) ( $\bullet$ Tab. 3) [5]. Weder Laborwerte noch apparative Diagnostik sind notwendig. Ist der Verdacht auf eine sekundäre Hyperhidrose gegeben (z.B. Nachtschweiß, unilaterale Hyperhidrose), so sind entsprechende Durchuntersuchungen notwendig. Grundsätzlich sind keine speziellen qualitativen (z. B. Jod-Stärke-Test nach Minor) oder quantitativen (z.B. Gravimetrie) Testverfahren in der klinischen Routine notwendig $[1,2]$. Jedoch können diese vorteilhaft sein, um Therapieverläufe zu dokumentieren, Kostenübernahmen bei der Krankenkasse zu beantragen oder im Rahmen von wissenschaftlichen Studien [6]. Als Nachteil aller aktuell verfügbaren Testverfahren gilt die zeitlich punktuelle Erfassung der Sekretion, die dem situativen Charakter der Hyperhidrose nicht immer gerecht wird.

\section{Allgemeine Überlegungen zur Therapie $\nabla$}

Eine fokale Hyperhidrose wird initial konservativ therapiert, ggf. auch durch Kombination verschiedener Verfahren [1,2,5]. Erst bei Versagen konservativer Maßnahmen sollte die Indikation zur Operation gestellt werden [6]. Operative Verfahren in der Behandlung der Hyperhidrose, mit Ausnahme von Eingriffen am Sympathikus, sind ureigenes Kerngebiet der Dermatochirurgie. Dermatologen haben in der Vergangenheit die entsprechenden Techniken maßgeblich geprägt und wissenschaftlich aufgearbeitet. Daher scheint es von Bedeutung, als Dermatochirurg fundierte Kenntnisse über operative Maßnahmen in der Behandlung der Hyperhidrose zu besitzen. Hieraus 
Tab. 1 Anamnestische Angaben bei primärer fokaler Hyperhidrose. Modifiziert nach Wörle et al. J Dtsch Dermatol Ges 2007.

Beginn im Kindes- oder Jugendalter (<25 Jahre)
Schwitzen ist temperaturunabhängig, unvorhersehbar, nicht willentlich
kontrollierbar
Symmetrisches fokales Auftreten an Prädilektionsstellen
(z. B. axillär, palmoplantar)
Auftreten öfter als $1 \times /$ Woche mit Beeinträchtigung des Allgemein-
befindens
Kein vermehrtes Schwitzen während der Schlafphasen
Positive Familienanamnese

Tab.2 Klinische Einteilung des Schweregrades der primären axillären (AH) und palmoplantaren (PH) Hyperhidrose. Modifiziert nach Wörle et al. J Dtsch Dermatol Ges 2007.

\begin{tabular}{|c|c|}
\hline $\begin{array}{l}\text { Grad I } \\
\text { Leichte } \\
\text { Hyperhidrose }\end{array}$ & $\begin{array}{l}\text { AH + PH: Deutlich vermehrte Hautfeuchtigkeit } \\
\mathrm{AH} \text { : Schwitzflecke (5-10 cm Durchmesser) }\end{array}$ \\
\hline $\begin{array}{l}\text { Grad II } \\
\text { Mäßig starke } \\
\text { Hyperhidrose }\end{array}$ & $\begin{array}{l}\text { AH + PH: Bildung von Schweißperlen } \\
\text { AH: Schwitzflecken ( } 10-20 \mathrm{~cm} \text { Durchmesser) } \\
\text { PH: Schwitzen auf Palmae und Plantae begrenzt }\end{array}$ \\
\hline $\begin{array}{l}\text { Grad III } \\
\text { Starke } \\
\text { Hyperhidrose }\end{array}$ & $\begin{array}{l}\text { AH + PH: Schweiß tropft ab } \\
\text { AH: Schwitzflecken (> } 20 \mathrm{~cm} \text { Durchmesser) } \\
\mathrm{PH}: \text { Schwitzen auch an dorsalen Fingern und Zehen } \\
\text { sowie am seitlichen Rand von Hand und Fuß }\end{array}$ \\
\hline
\end{tabular}

Tab.3 Hyperhidrosis Disease Severity Scale (HDSS). Modifiziert nach Lowe et al. J Am Acad Dermatol 2007. ${ }^{1}$

Grad 1: Schwitzen ist stets unauffällig und beeinträchtigt meine
Alltagsaktivitäten nie
Grad 2: Schwitzen ist erträglich, beeinträchtigt jedoch gelegentlich
meine Alltagsaktivitäten
Grad 3: Schwitzen ist kaum erträglich und beeinträchtigt häufig meine
Alltagsaktivitäten
Grad 4: Schwitzen ist unerträglich und beeinträchtigt meine Alltags-
aktivitäten fortwährend
1 Ein HDSS-Wert von 3 oder 4 gilt als Hinweis auf eine starke Ausprägung der Erkran-
kung.

erwächst auch die besondere Verantwortung und Pflicht, unseren Patienten eine stadiengerechte Therapie anzubieten, und nicht den Weg des vorschnellen, oft lukrativen „Griffs zum Messer" einzuschlagen.

\section{Operationsverfahren}

$\nabla$

Eingriffe können in lokal chirurgische Techniken und Operationen direkt am Sympathikus eingeteilt werden ( $\bullet$ Tab.4) [6]. Die anatomischen Gegebenheiten bedingen, dass lokal chirurgische Verfahren nur im axillären Bereich durchgeführt werden können. Die palmoplantare Region ist hierfür nicht zugänglich. Alle Operationen an der axillären Haut können problemlos in Lokalanästhesie bzw. Tumeszenzanästhesie erfolgen, falls notwendig mit Sedierung des Patienten [6]. Sympathektomien benötigen hingegen eine spezielle Intubationsnarkose, da die Lunge kollabiert werden muss, um den Sympathikus im Bereich der Ganglien T2T4 unterbrechen zu können $[1,7]$. Heutzutage wird der Eingriff endoskopisch durchgeführt (endoskopisch-transthorakale Sympathektomie/ETS), die klassische offene Sympathektomie ist obsolet.
Tab. 4 Chirurgische Techniken zur Therapie primärer fokaler Hyperhidrosen.
I. Lokal chirurgische Verfahren
I.1. KomSplette Exzision
I.2. Teilexzision mit offener Adenektomie
I.3. Subkutane Verfahren
II. Eingriffe am Sympathikus
II.1. Endoskopisch-transthorakale Sympathektomie (ETS)
II.2. Computertomografie-gesteuerte Sympathikolyse

\section{Kritische Anmerkungen}

Bei genauer Sichtung der Literatur zu operativen Verfahren fällt auf, dass eine Reihe von Studien mit großen Patientenkollektiven aus dem asiatischen Raum stammt. Häufige Operationsindikation dieser Untersuchungen ist die in der asiatischen Kultur stark stigmatisierende Bromhidrose/Osmidrose (Geruchsschwitzen), die als apokrin induziert angesehen wird [8-10]. Histologisch betrachtet, liegen die apokrinen Schweißdrüsen vorwiegend im superfiziellen subkutanen Fettgewebe an der Dermis-FettGrenze, wohingegen die ekkrinen Drüsen v.a. im tiefen Korium liegen [1]. Die Operationsergebnisse der Bromhidrose können daher nicht direkt auf die durch eine erhöhte Stimulation der ekkrinen Drüsen ausgelöste Hyperhidrose übertragen werden, da die subkutane Entfernung ekkriner Drüsen technisch schwieriger ist. Eine Vermischung von Studien zur Behandlung der Bromhidrose und Hyperhidrose ist jedoch in vielen Publikationen zu beobachten, obwohl hierdurch ein fehlerhafter Vergleich von Effizienz und Komplikationsraten der Operationsverfahren entsteht.

\section{Lokal chirurgische Techniken \\ $\nabla$}

\section{Komplette Exzision}

Ziel ist die Exzision des gesamten hyperhidrotischen Areals. Bei kleineren Arealen ist dies oft komplikationsarm möglich, wohingegen größere Defekte meist durch Z-Plastiken oder Transpositions-Lappen gedeckt werden müssen [6]. Um sich ein Bild über das hyperhidrotische Areal machen zu können, erscheinen präoperativ qualitative Tests, ggf. auch repetitiv, sinnvoll. Der Vorteil der Methode ist die große Wirksamkeit, nachteilig sind die möglichen Nebenwirkungen der Lappenplastiken (Dehiszenz, Hautnekrose, Wundinfektion, Narbenkontraktur). Der Verschluss mittels Z-Plastik nach Bretteville-Jensen zeigte in einer Studie an 123 Patienten mit axillärer Hyperhidrose eine Erfolgsrate von 94,3\% [11]. Die ausgedehnten Resektionstechniken werden heutzutage selten durchgeführt.

\section{Teilexzision mit offener Adenektomie}

Es werden nur die zentralen hyperhidrotischen Bereiche spindeloder S-förmig exzidiert $[12,13]$. Im Anschluss wird die umgebende Haut nach Unterminierung aufgeklappt und die Drüsen werden unter Sicht mittels Schere abgetragen $(\boldsymbol{A}$ Abb.1). Durch die geringere Menge an resezierter Haut soll die Rate an Nekrosen, Nahtdehiszenzen und hypertrophen Narben und Keloiden reduziert werden. In einer Arbeit von Lawrence zeigte sich bei spindelförmiger Exzision (Verfahren nach Shelley) nach einer durchschnittlichen Nachbeobachtungszeit von 1,3 Jahren eine Reduktion der Schweißmenge um 65\%. An Nebenwirkungen traten Hämatome in $26 \%$ und Wundinfekte in 13\% auf [14] 


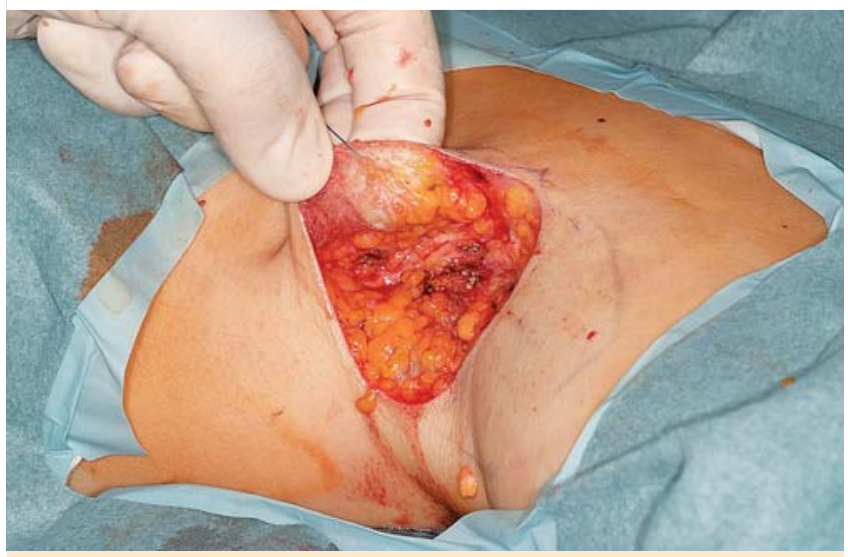

Abb. 1 Intraoperatives Bild bei zentraler Teilexzision nach Shelley. Nach Exzision wird der umgebende Bereich unterminiert. Im Anschluss werden die Schweißdrüsen unter Sicht entfernt.

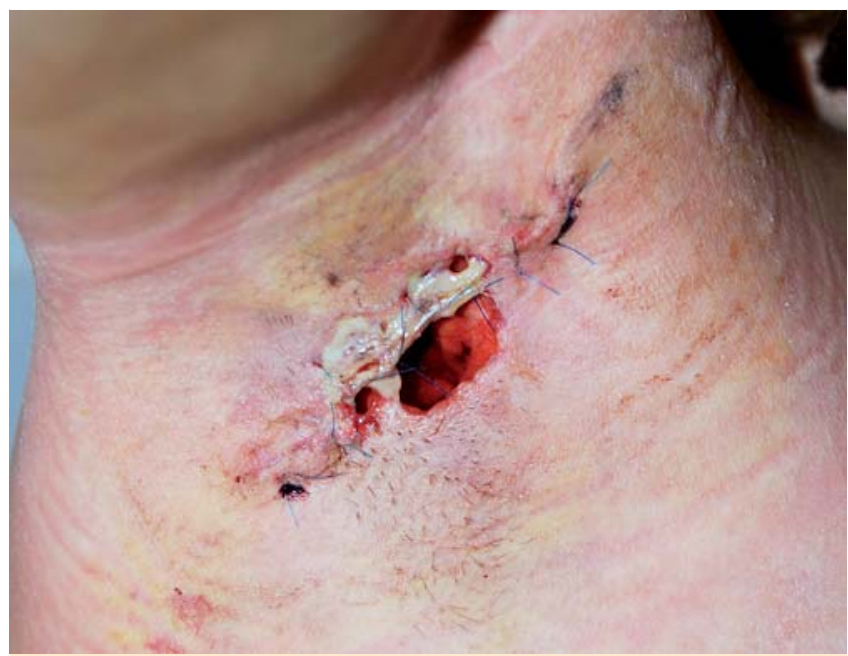

Abb.2 Nahtdehiszenz und kleine Hautnekrose nach Teilexzision. 8 Tage postoperativ.

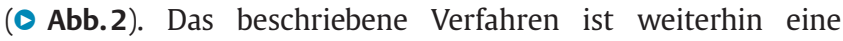
Standardtechnik in der operativen Therapie der axillären Hyperhidrose ( $\bullet$ Abb.3 u. $\bullet$ Abb.4).

\section{Subkutane Verfahren}

Die Techniken haben zum Ziel, Schweißdrüsen zu entfernen oder zu destruieren, ohne die darüber liegende Haut zu resezieren. In den letzten Jahren ist dieser Teilbereich der operativen Techniken durch immer neue Ansätze und Instrumente erweitert worden, von denen jedoch viele eher als eminenzbasiert und nicht evidenzbasiert eingestuft werden müssen.

\section{Subkutane Kürettage/Shaving}

Seit Jahrzehnten ist die subkutane Kürettage (Syn.: manuelles Shaving, geschlossene Kürettage) ein fester Bestandteil des operativen Spektrums. In der Regel wird nach Unterminierung der axillären Haut versucht, mit einer gynäkologischen Kürette Schweißdrüsen abzuschaben [6]. Alternativ zur gynäkologischen Kürette kann das Verfahren mittels dermaler Ring-Kürette, einem scharfen Löffel oder speziell entwickeltem Instrumentarium durchgeführt werden [6]. In der Erstbeschreibung von Jemec wurden 168 Patienten mit axillärer Hyperhidrose behandelt. Die Rezidivquote lag bei $15 \%$, die Nebenwirkungsrate wurde

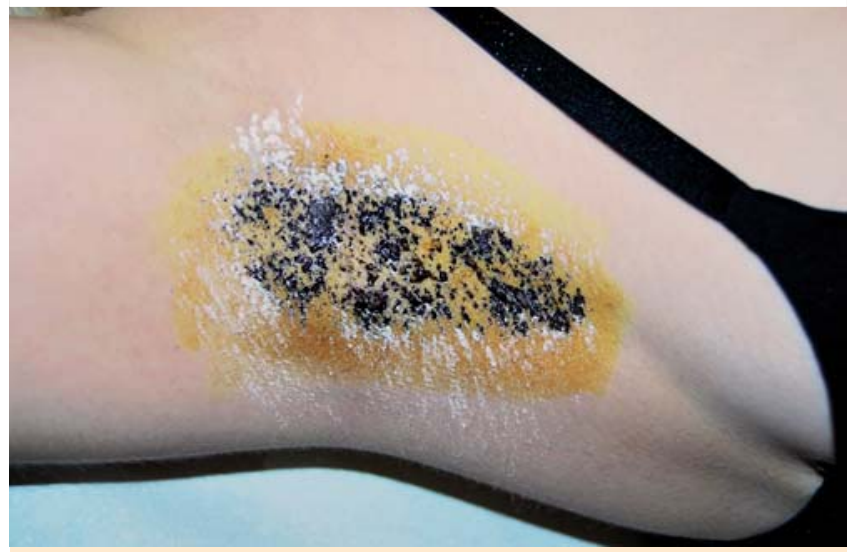

Abb.3 Hyperhidrotisches Areal der rechten Axilla bei einer 25-jährigen Patientin. Die gelbliche Färbung entsteht nach Auftragen der Jod-Lösung. Das violett-blaue Areal identifiziert den hyperhidrotischen Bereich durch eine Jod-Stärke-Reaktion nach Auftragen von Speisestärke (Minor-Test).

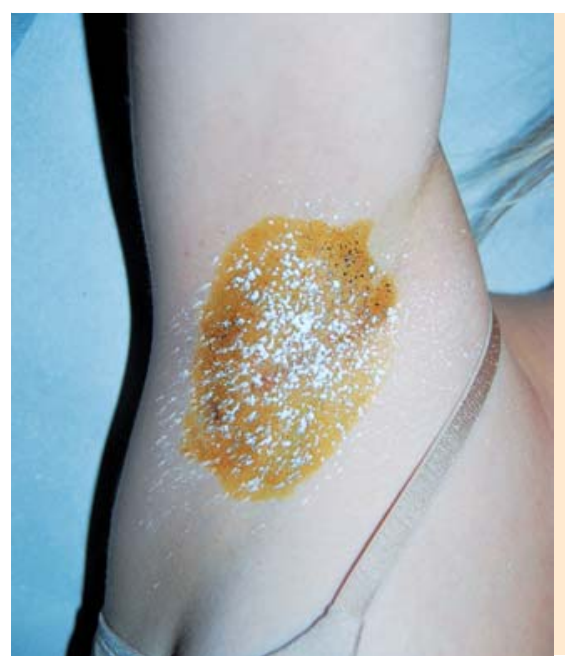

Abb.4 Zwölf Monate nach zentraler Teilexzision und kombinierter offener Adenektomie. Deutliche Reduktion des hyperhidrotischen Bereichs im Vergleich zu - Abb. 3.

mit 35\% angegeben (z.B. Hautnekrosen, Hämatome, Lymphfisteln) [15]. Alternativ können elektrisch betriebene Geräte zur Ablation der Drüsen eingesetzt werden. Beispielhaft seien Arthroskopie-Instrumente aus der orthopädischen Chirurgie (sog. Knorpel-Shaver) genannt. Die Erfolgsquote scheint hierbei hoch zu sein, so konnten Arneja et al. bei 50 Patienten nach einer durchschnittlichen Nachbeobachtungszeit von 28 Monaten eine suffiziente Reduktion der Hyperhidrose bei $94 \%$ der Behandelten aufzeigen [16]. Einschränkend sind jedoch der hohe apparative Aufwand und die Tatsache, dass die orthopädisch-chirurgischen Instrumente nicht zur Standardausrüstung der Dermatochirurgie gehören.

\section{Subkutane Laser-Verfahren}

Nach Dissektion von Haut und Fettgewebe wird eine Laserfaser unter das hyperhidrotische Areal eingeführt. Studien zur Benutzung einer $\mathrm{CO}_{2}$-Laser-Faser (10600 nm) gibt es lediglich für die Indikation Bromhidrose/Osmidrose [10]. Die Nebenwirkungsrate (v.a. Hautnekrosen und Verbrennungen) scheint stark von der Erfahrung des Operateurs abzuhängen. Über positive Erfahrungen bei der Verwendung einer flexiblen $1064 \mathrm{~nm}$-Nd:YAG Laserfaser berichten Goldman und Wollina [17]. Aktuell gibt es, bedingt durch den Vormarsch der Laser-Lipolyse zur Fettreduktion, Ansätze, mit verschiedenen Wellenlängen eine Destruktion von Schweißdrüsen herbeizuführen. Gängige Wellenlängen der 


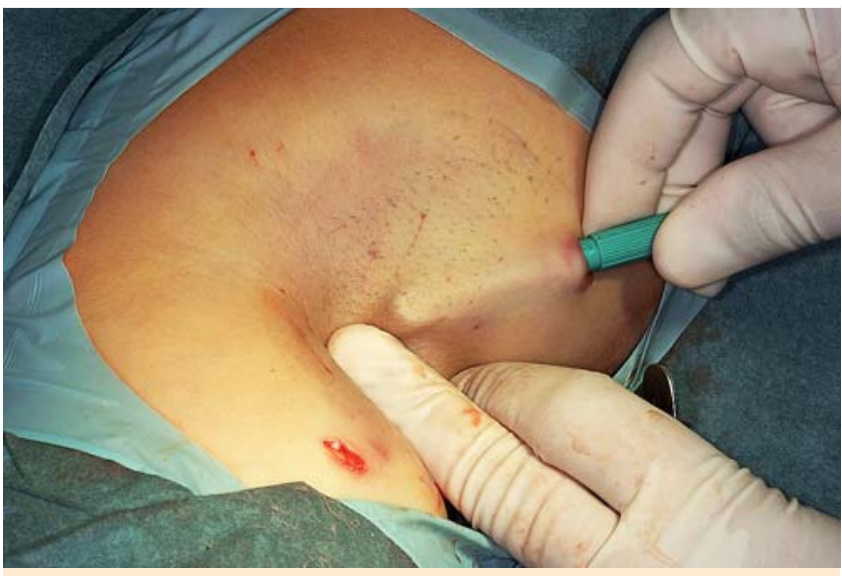

Abb.5 2. Operationsschritt bei einer Schweißdrüsen-Saugkürettage. Nach Absaugung erfolgt eine subkutane Kürettage mittels dermaler RingKürette. CAVE: Es kann hierbei leicht zur Perforation der Haut kommen.

derzeitig verfügbaren Geräte sind 924, 975, 1064, 1320 und 1440 $\mathrm{nm}$. Obwohl in der Theorie der Einsatz der Laser-Lipolyse in der Behandlung der axillären Hyperhidrose interessante Ansätze zu haben scheint, gibt es bislang bis auf einzelne Fallberichte keine Studiendaten, die eine Effektivität belegen [18]. Es bleibt daher abzuwarten, ob sich das System in der Zukunft bewährt. Aktuell ist die Technik lediglich als experimenteller Ansatz zu werten.

\section{Schweißdrüsen-Saugkürettage}

Die Technik kombiniert eine superfizielle Liposuktion mit einer subkutanen Kürettage [19-21]. Die Dissektion von Subkutis und Haut kann stumpf mit der Kanüle im Rahmen der Liposuktion erfolgen. Bei Benutzung einer stumpfen Kanüle erfolgt die anschließende Kürettage der Dermis-Fett-Grenze mittels scharfem Löffel oder einer Kürette ( $\bullet$ Abb.5). Das gelöste Gewebe wird im Anschluss aspiriert. Bei Benutzung einer speziellen scharfen Kanüle erfolgen Absaugung und Kürettage mit einem Instrument. Scharfe Kanülen scheinen effektiver im Vergleich zu stumpfen Liposuktionskanülen zu sein [20]. Eine Studie konnte 1 Jahr postoperativ einen Rückgang der Schweißmenge von 66,4\%, bei gleichzeitig histologisch gesicherter Reduktion der Schweißdrüsendichte von 79,1\% aufzeigen [19]. Neben der reduzierten Schweißmenge konnte nach erfolgreicher Operation auch eine Besserung der Lebensqualität bei den Behandelten erzielt werden [4]. Nebenwirkungen wie Hämatome, Serome, Dysästhesien, subkutane Strangbildung und Hautnekrosen sind beschrieben [6] (๑ Abb.6 u. $\odot$ Abb.7).

\section{Eingriffe am Sympathikus \\ $\nabla$}

\section{Endoskopisch-transthorakale Sympathektomie (ETS)}

Bei der ETS wird der Grenzstrang vom zweiten bis zum fünften thorakalen Ganglion reseziert, koaguliert, mit Radiofrequenz verödet oder mittels Metallclip unterbrochen [7]. Das endoskopische Verfahren wird meist von Thorax- oder Gefäßchirurgen in Intubationsnarkose durchgeführt. Da bei palmarer Hyperhidrose kein lokal chirurgisches Verfahren möglich ist, stellt die ETS die Ultima Ratio bei schwerster therapieresistenter Form dar. Die Erfolgsraten sind mit bis zu 95\% sehr hoch [22,23]. Da für die axilläre Hyperhidrose effiziente lokal-chirurgische Techniken existieren und die Erfolgsraten der ETS geringer als bei der pal-
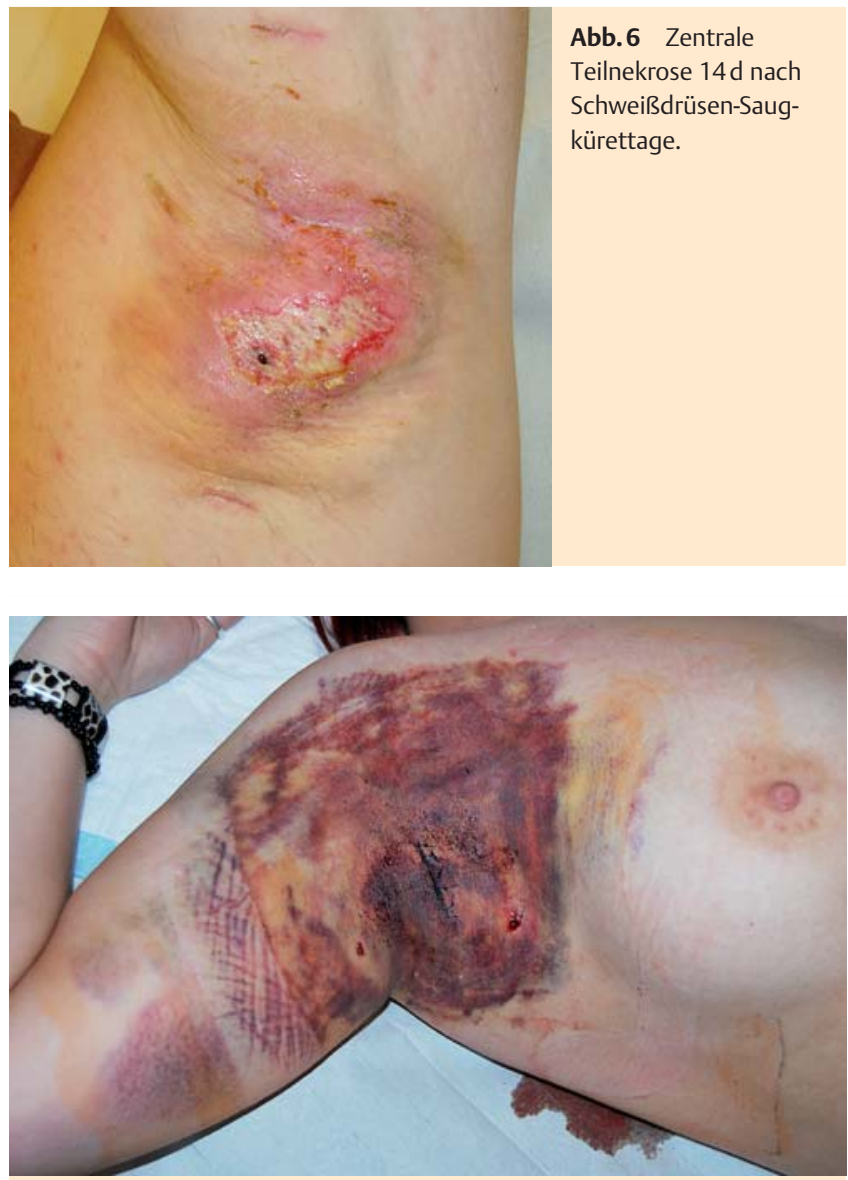

Abb.7 Ausgedehntes Hämatom nach Schweißdrüsen-Saugkürettage. Operative Revision mit Ausräumung des Hämatoms wurde notwendig.

maren Hyperhidrose zu sein scheinen, ist die ETS hierbei nicht indiziert. Neben den allgemeinen Operations-Risiken (v.a. Pneumothorax und Interkostalneuralgien) ist als postoperative Komplikation das Auftreten eines Horner-Syndroms bei Verletzung des ersten thorakalen Ganglions beschrieben. Typische Nebenwirkungen für die ETS sind darüber hinaus ein postoperatives gustatorisches Schwitzen (=fokale Hyperhidrose meist im Gesicht bei Nahrungsaufnahme) sowie je nach Studienlage in bis zu 90\% ein kompensatorisches Schwitzen (v.a. am Rumpf und den proximalen Oberschenkeln) [22,23]. Die Ausprägung des kompensatorischen Schwitzens soll von der Höhe des ausgeschalteten Ganglions abhängen [7].

\section{Computertomografie-gesteuerte Sympathikolyse}

Hierbei wird versucht, eine Destruktion der Ganglien durch eine perkutane Injektion von konzentriertem Alkohol herbeizuführen. Neben der palmaren Hyperhidrose soll die Technik auch bei plantarer Hyperhidrose einsetzbar sein. Die Studienlage ist für das Verfahren deutlich schlechter als für die ETS [24]. Neuerdings wurde auch die magnetresonanz-tomografisch (MRT)-gesteuerte Sympathikolyse beschrieben [25].

\section{Zusammenfassung und Ausblick}

$\nabla$

Operative Verfahren sind eine wichtige Säule in der Therapie therapierefraktärer fokaler Hyperhidrosen. Der erfahrene Dermatochirurg fungiert als kompetenter Ansprechpartner, der 
diagnostisch und therapeutisch das gesamte Spektrum des Krankheitsbildes abdeckt. Trotz der in den letzten Jahren zunehmend besseren Datenlage zur Effektivität, gibt es bislang keine ausreichende Studienlage, um einen operativen Goldstandard festzulegen. Erstaunlich ist, dass bislang keine einzige Seitenvergleichs-Studie existiert. Um diese Lücke zu schließen, wurde unter der Leitung des Autors eine Multizenter-Studie initiiert, mit dem Ziel im Seitenvergleich bei 100 Patienten die klassische Resektionstechnik nach Shelley mit der Schweißdrüsen-Saugkürettage zu vergleichen. Die Ergebnisse der 2-jährigen Nachbeobachtung dieser gravimetrisch-kontrollierten Studie werden möglicherweise dazu beitragen, zukünftig den Stellenwert der einzelnen lokal-chirurgischen Techniken exakter zu definieren.

\section{Interessenkonflikt}

$\nabla$

Der Autor gibt an, dass kein Interessenkonflikt besteht.

\section{Abstract}

\section{Surgical Treatment for Hyperhidrosis}

Surgical therapy is the ultima ratio for the treatment of hyperhidrosis after conservative procedures have failed. Surgery is only suitable for focal hyperhidrosis. Local surgical procedures are preserved for axillary hyperhidrosis. Endoscopic sympathectomy is a therapy option for severe palmar hyperhidrosis. However, for most surgical treatments evidence-based data concerning efficacy and long-term results are missing.

\section{Literatur}

1 Bechara FG, Schmidt J, Hoffmann K, Altmeyer P. Krankhaftes Schwitzen. Stuttgart: Kohlhammer; 2008

2 Wörle B, Rapprich S, Heckmann M. Definition and treatment of primary hyperhidrosis. J Dtsch Dermatol Ges 2007; 5: 625-628

3 Strutton DR, Kowalski JW, Glaser DA et al. US prevalence of hyperhidrosis and impact on individuals with axillary hyperhidrosis: Results from a national survey. J Am Acad Dermatol 2004; 51: 241 -248

4 Bechara FG, Gambichler T, Sand M et al. Assessment of quality of life in patients with axillary hyperhidrosis before and after suction-curettage. J Am Acad Dermatol 2007; 57: 207-212

5 Lowe NJ, Glaser DA, Eadie N et al. Botulinum toxin type A in the treatment of primary axillary hyperhidrosis: a 52-week multicenter dou- ble-blind, randomized, placebo-controlled study of efficacy and safety. J Am Acad Dermatol 2007; 56: 604-611

6 Bechara FG. Aktuelle Therapie der fokalen Hyperhidrose. Operative Verfahren. Hautarzt 2009; 60: 538-545

7 Schmidt J, Bechara FG, Altmeyer P et al. Endoscopic thoracic sympathectomy for severe hyperhidrosis: Impact of restrictive denervation on compensatory sweating. Ann Thorac Surg 2006; 81: 1048-1055

8 Huang $\mathrm{YH}$, Yang $\mathrm{CH}$, Chen $\mathrm{YH}$ et al. Reduction in osmidrosis using a suction-assisted cartilage shaver improves the quality of life. Dermatol Surg 2010; 36: 1573-1577

9 Liu $Q$ Zhou $Q$, Song Y et al. Surgical subcision as a cost-effective and minimally invasive treatment for axillary osmidrosis. J Cosmet Dermatol 2010; 9: 44-49

$10 \mathrm{Kim} \mathrm{ICH}$, Seo SL, Oh $\mathrm{CH}$. Minimally invasive surgery for axillary osmidrosis: Combined operation with $\mathrm{CO} 2$ laser and subcutaneous tissue remover. Dermatol Surg 1999; 25: 875-879

11 Bretteville-Jensen $G$. Radical sweat gland ablation for axillary hyperhidrosis. Br J Plast Surg 1973; 26: 158-162

12 Hurley HJ, Shelley WB. Axillary hyperhidrosis. Clinical features and local surgical management.. Br J Dermatol 1966; 78: 127-140

13 Bisbal J, del Cacho C, Casalots J. Surgical treatment of axillary hyperhidrosis. Ann Plast Surg 1987; 18: 429-436

14 Lawrence CM, Lonsdale Eccles AA. Selective sweat gland removal with minimal skin excision in the treatment of axillary hyperhidrosis: A retrospective clinical and histological review of 15 patients. Br J Dermatol 2006; 155: 115-118

15 Jemec B. Abrasio axillae in hyperhidrosis. Scand J Plast Reconstr Surg 1975; 9: 44-46

16 Arneja JS, Hayakawa TE, Singh GB et al. Axillary hyperhidrosis: A 5-year review of treatment efficacy and recurrence rates using a new arthroscopic shaver technique. Plast Reconstr Surg 2007; 119: 562-567

17 Goldman A, Wollina $U$. Subdermal Nd-YAG laser for axillary hyperhidrosis. Dermatol Surg 2008; 34: 756-762

18 Kotlus BS. Treatment of refractory axillary hyperhidrosis with a 1320nm Nd:YAG laser. J Cosmet Laser Ther 2011; 13: 193-195

19 Bechara FG, Sand M, Hoffmann K et al. Histological and clinical findings in different surgical strategies for focal axillary hyperhidrosis. Dermatol Surg 2008; 34: 1001 - 1009

20 Bechara FG, Sand M, Sand D et al. Suction-curettage as a surgical treatment of focal axillary hyperhidrosis: Recommendation for an aggressive approach. Plast Reconstr Surg 2007; 119: 1390-1391

21 Bechara FG, Sand M, Tomi NS et al. Repeat liposuction-curettage is safe and effective. Br J Dermatol 2007; 157: 739-743

22 Dumont $P$, Denoyer A, Robin P. Long-term results of thoracoscopic sympathectomy for hyperhidrosis. Ann Thorac Surg 2004; 78: 1801 - 1807

23 Gossot D, Galetta D, Pascal A et al. Long-term results of endoscopic thoracic sympathectomy for upper limb hyperhidrosis. Ann Thorac Surg 2003; 75: 1075-1079

24 Schneider B, Richter GM, Roeren T et al. CT-gesteuerte Neurolysen. Stand der Technik und aktuelle Ergebnisse. Radiologe 1996; 9: 692 699

25 Konig CW, Schott UG, Pereira PL et al. MR-guided lumbar sympathicolysis. Eur Radiol 2002; 12: 1388-1393 